\title{
Activating NTRK Family Point Mutation
}

National Cancer Institute

\section{Source}

National Cancer Institute. Activating NTRK Family Point Mutation. NCI Thesaurus. Code C150439.

A point mutation in an NT RK family gene that causes the encoded receptor tyrosine kinase and its downstream pathways to be constitutively activated. 